\title{
A Synopsis of Pterostemon (Iteaceae), a Group Endemic to Mexico
}

\author{
Judith Márquez Guzmán ${ }^{1}$, Martha Martínez Gordillo², Ramiro Cruz Durán ${ }^{2 *}$, \\ Jaime Jiménez Ramírez ${ }^{2}$, María Cristina Pérez-Amador ${ }^{3}$ \\ ${ }^{1}$ Laboratorio de Desarrollo en Plantas, Facultad de Ciencias, Universidad Nacional Autónoma de México, México D. F., México; \\ ${ }^{2}$ Departamento de Biología Comparada, Facultad de Ciencias, Universidad Nacional Autónoma de México, México D.F., México; \\ ${ }^{3}$ Laboratorio de Química, Facultad de Ciencias, Universidad Nacional Autónoma de México, México D. F., México. \\ Email: rramcrudur@yahoo.com
}

Received July $22^{\text {nd }}, 2013$; revised August $22^{\text {nd }}, 2013$; accepted September $25^{\text {th }}, 2013$

Copyright (C) 2013 Judith Márquez Guzmán et al. This is an open access article distributed under the Creative Commons Attribution License, which permits unrestricted use, distribution, and reproduction in any medium, provided the original work is properly cited.

\begin{abstract}
The genus Pterostemon Schauer (Iteaceae), is a group of three species restricted in distribution to Mexico. It includes: Pterostemon mexicanus Schauer, P. rotundifolius Ramírez, and P. bravoanus J. Jiménez-Ram. \& Martínez Gordillo. On the basis of field observations and herbarium specimens, we review the morphology (vegetative and reproductive), and distribution to give an updated taxonomic treatment for the genus. Additionally the utility of morphological characters is evaluated for taxomic use, and detailed taxonomic descriptions and ecological associations are given for each species.
\end{abstract}

Keywords: Endemic; Mexico; Pterostemon; Iteaceae

\section{Introduction}

Pterostemon Schauer is a small genus of three species endemic to arid and semi-arid regions of central and southern Mexico [1]. Schauer (1847) [2] first described Pterostemon mexicanus. Schauer placed this species in its own family, Pterostemonaceae. Since then the position of the genus has been questioned and it has been placed in different families including Saxifragaceae by Baillon (1872) [3], Engler and Prantl (1928) [4], Erdtman (1952) [5] and Schulze-Menz (1964) [6], Grossulariaceae by Cronquist (1981) [7], and Escalloniaceae by Thorne (1992) [8]. Small (1905) [9], Hutchinson (1967) [10], Dahlgren (1989) [11], Takhtajan (1988) [12], and PérezCálix (2003) [13] and the Angiosperm Phylogeny Group (2009) [14] support its placement as the only genus in the family Pterostemonaceae, as first proposed by Schauer (1847). Embryological and phylogenetic analyses [15-17] have provided additional evidence in support of the recognition of the family Pterostemonaceae which has been shown to be closely related to the Iteaceae; nevertheless, according to the Angiosperm Phylogeny Group o APG III (2009) classification, Pterostemon is included into the

"Corresponding author. family Iteaceae.

Ptersotemon is distinguished primarily by the combination of its woody habit, pubescent stems with lenticels, leaves with stipules, production of resin, the presence of a pubescent hypanthium, 10 stamens ( 5 fertile and 5 staminodia) with flattened filaments and toothed apices, and the capsular fruit containing one or rarely two seeds with endosperm. The most important characters to separate different species are the shape of the leaves, the size of the petioles, the number of flowers per inflorescences, the petals and their position during anthesis.

Natural populations of the three species of Pterostemon are usually small and highly localized, and even though usually a large proportion of flowers can be seen, in at least one of the species the production of seeds is very scarce and the establishment of seedlings is over all very low.

\section{Material and Methods}

In order to review and consolidate the taxonomic information available for the genus, herbarium material was reviewed from the herbaria ENCB, FCME, IEB, MEXU, MO, TEX, and XAL. In addition, field work was conducted to allow for collection of fresh material as well as 
recording of direct observations of the species in the wild. The field work was conducted in the states of Puebla, Hidalgo, Oaxaca, and Guerrero. Plant material was collected for further analysis under the Scanning Electron Microscope, phytochemical as well as embryological studies. For all the species in the genus, a taxonomic description, as well as phenological and environmental data are included.

\section{Results}

\subsection{The Genus Pterostemon}

Pterostemon Schauer, Linnaea 20:736. 1847. Type: Pterostemon mexicanus Schauer, Linnaea 20: 736. 1847. crescit in montosis Mexici, ad Zimapan, Aschb. 259.

Shrubs or small trees; stems cylindrical; cortex fissured, gray or maroon, smooth or ribbed, glabrescent, lenticels present, maroon or dark gray; indumentum of simple trichomes, generally with a base of $5-7$ cells, sometimes very conspicuous, usually papillated (Figure 1), and with spherical glands (Figures 2-7). Leaves patiolate, reddish with age, hypostomatic; petioles usually pubescent; stipules small, usually deciduous; blades orbiculate, sub-orbiculate, oblog-orbiculate, widely elliptic, obovate or widely obovate, membranaceous or coriaceous, apex obtuse or rounded, sometimes notched, margin crenated-toothed or toothed, sometimes revolute, glandular teeth, base rounded or cuneiform, sometimes slightly oblique or subcordate; adaxial surface green, pubescent or glabrous, abaxial surface pale, glabrous or pubescent; 3 - 6 pairs of secondary nerves. Inflorescence terminal or subterminal (Figure 8), peduncle and rachis usually pubescent, (1-)5 to more than 50 flowers; bracteoles small, pubescent, with spheric glands on the margins. Flowers generally pedicelate (Figure 9); pedicel pubescent; hypanthium conspicuous, pubescent; calyx with 5 sepals, connate at the base; lobules linear-lanceolate, deltate-lanceolate or deltate, erect, pubescent with spheric glands, valvate, persistent, adaxial surface pubescent or glabrous, abaxial surface pubescent, usually glandular; corolla with 5 petals, free, oblong or elliptic, apex usually rounded, sometimes emarginated, base unguiculate to straight, white or pink, adaxial surface glabrous or pubescent, abaxial surface pubescent, margin usually involute with erect or reflexed spherical glands; stamens 10,5 fertile, 5 staminodia, erect; filaments flattened, apex denticulate, pubescent on both sides; anthers glabrous, connective tissue apiculate (Figure 10); pollen tricolporoidate or tricolporate, bicellular or tricellular at the time the monads are freed; exine tectate, perforate, sometimes suprarrugulate. Gynoecium syncarpic, ovary inferior, 5(-6)-carpels, 4 - 8 ovules per locule; pubescent on the exterior surface, styles 5 , connate at different degrees, stigmas 5, lobed, glabrous; ovules crassinucelated, ana-

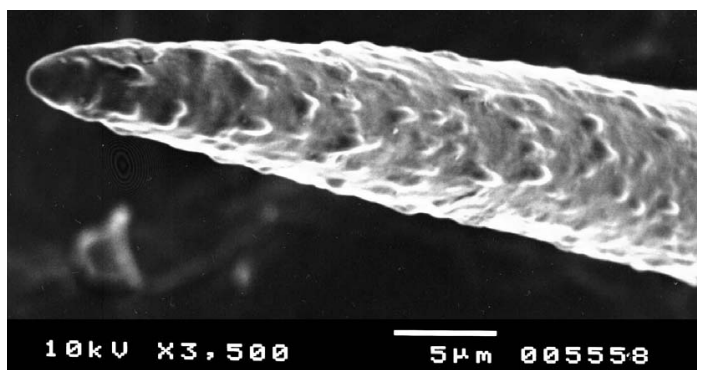

Figure 1. Surface of the trichome of Pterostemon bravoanus (from Cruz \& Ponce 5899 at FCME).

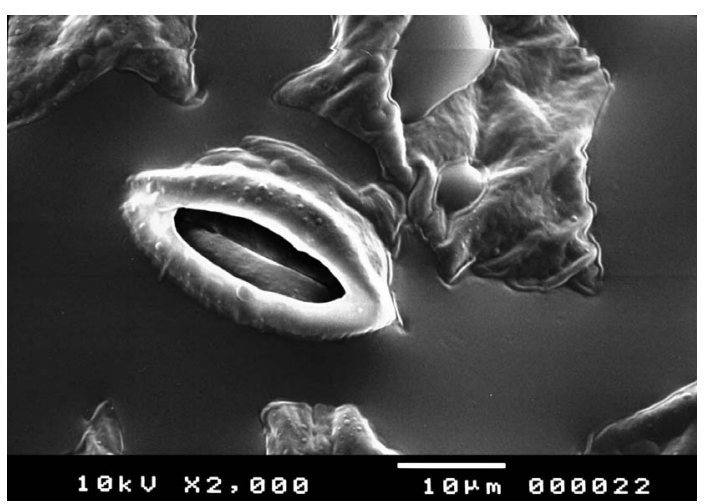

Figure 2. Stomata of Pterostemon rotundifolius (Vega 2 at FCME).

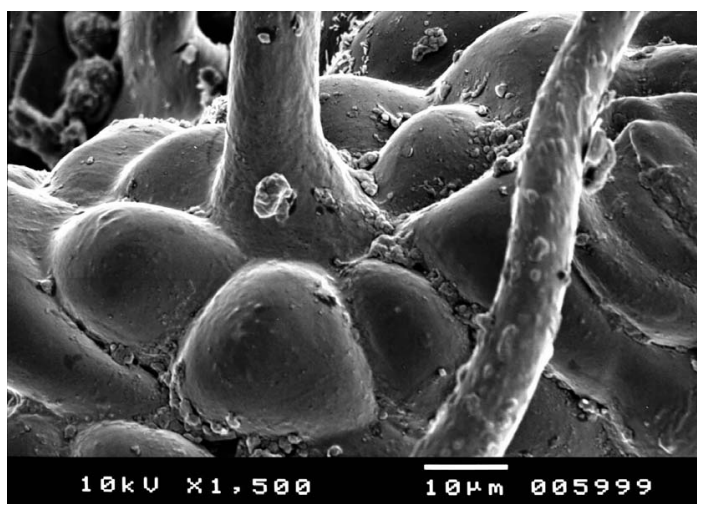

Figure 3. Cellules at the base of the trichomes of Pterostemon mexicanus, adaxial surface (from Cruz 5999 at FCME).

tropous, bitegmic; tegmens of epidermic origin, tristratified. Fruit an indehiscent capsule, floral whorls persistent (Figure 11). Seeds 1, rarely 2, ovoid, maroon.

Distribution and habitat. Pterostemon along with the family Iteaceae is a paleo-endemic restricted to Mexico. It is found in arid and semi-arid regions of central and southern Mexico. It comprises three species with narrow and scattered populations in the states of Querétaro, Hidalgo, Puebla, Oaxaca, Guerrero and San Luis Potosí. It can be found growing in well drained calcareous soils, in dry scrublands, tropical deciduous forest, Quercus forest, and Pinus and Juniperus forest between 500 and 2350 meters above sea level. 


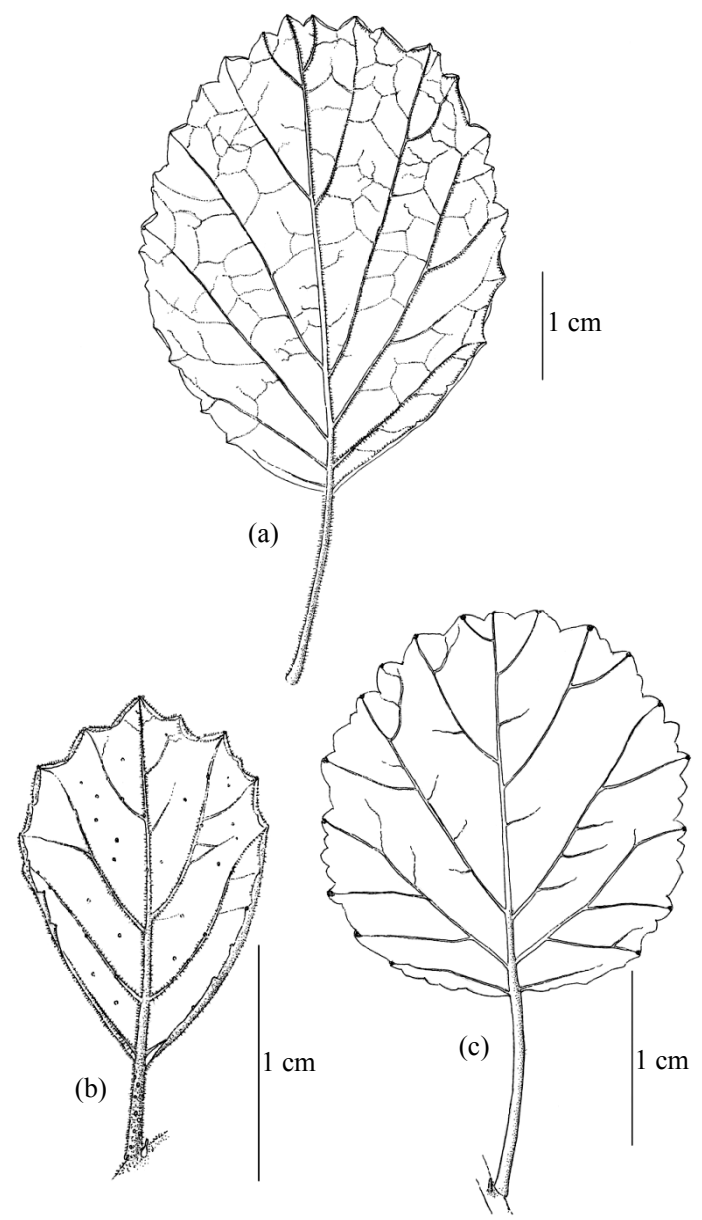

Figure 4. Leaves of Pterostemon. A. P. bravoanus (from Cruz \& Ponce 5899 at FCME). B. P. mexicanus (from Simón s.n. at FCME). C. P. rotundifolius (from Vega s.n. at FCME). Drawings R. Cruz.

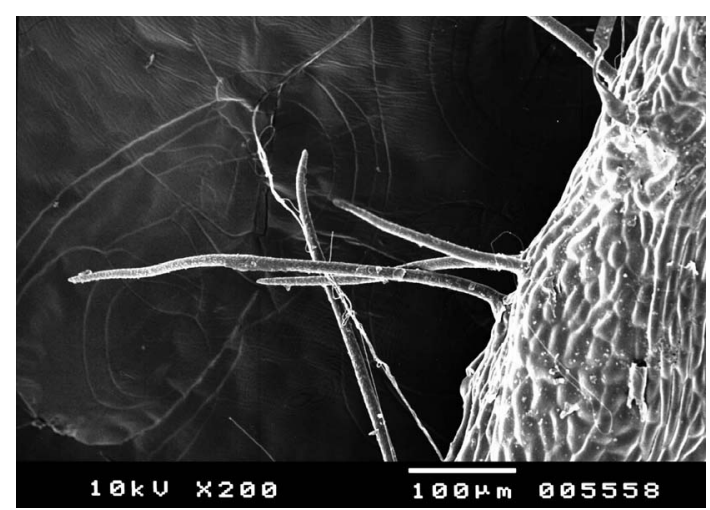

Figure 5. Margin of the leaf of Pterostemon bravoanus (from Cruz \& Ponce 5899 at FCME).

\subsection{Key to Species}

1) Leaves obovate, petiole 0.4 - $0.6 \mathrm{~cm}$ long; inflorescences usually with 20 flowers or less; Guanajuato, Querétaro, Hidalgo, San Luis Potosí, and Northern

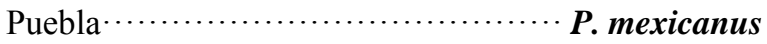

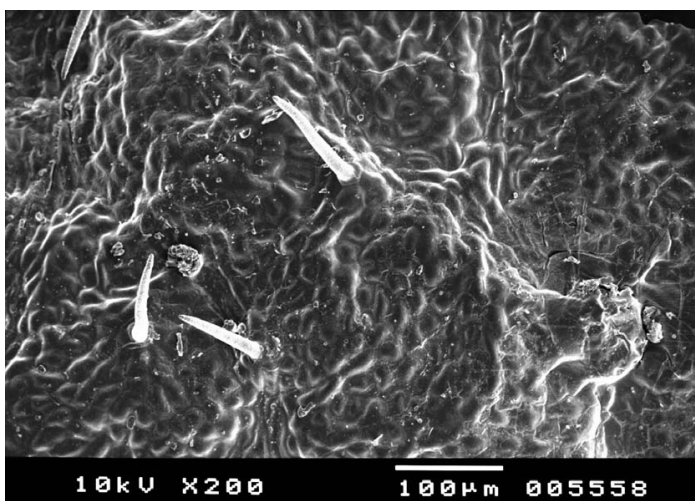

Figure 6. Exudates from glands in the adaxial surface of the leave of Pterostemon bravoanus (from Cruz \& Ponce 5899 at FCME).

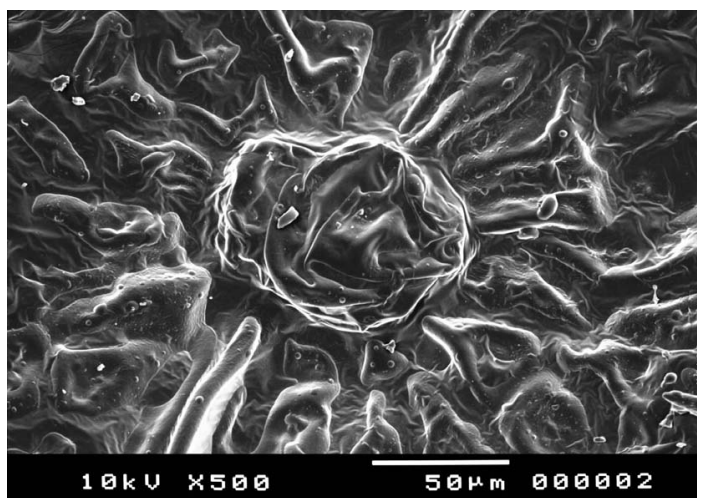

Figure 7. Spherical gland in the adaxial surface of the leaf of Pterostemon rotundifolius (from Vega 2 at FCME).

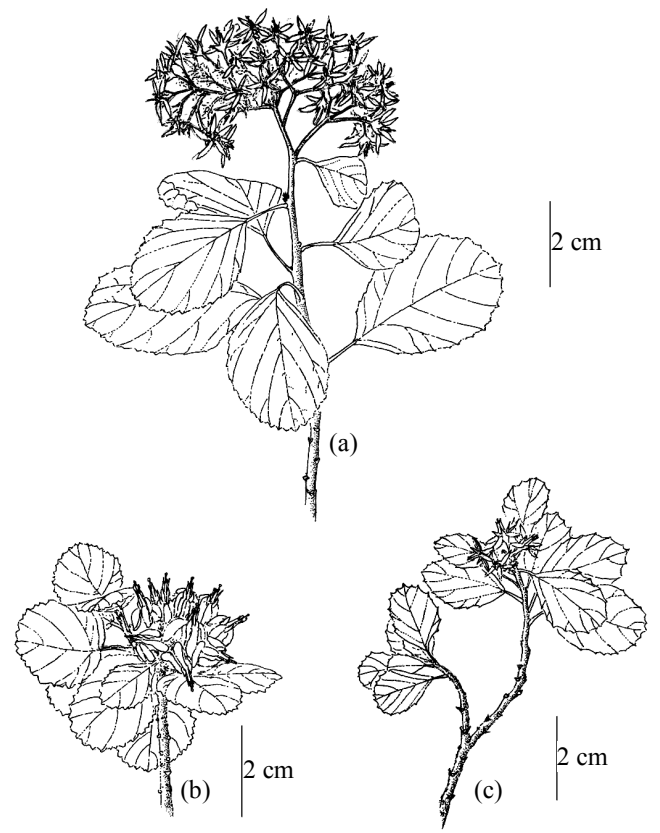

Figure 8. Inflorescences of Pterostemon. A. P. bravoanus (from Cruz \& Ponce 5899, FCME). B. P. rotundifolius (from Vega 3, FCME). C. P. mexicanus (from Cruz 5998, FCME). Drawings R. Cruz. 


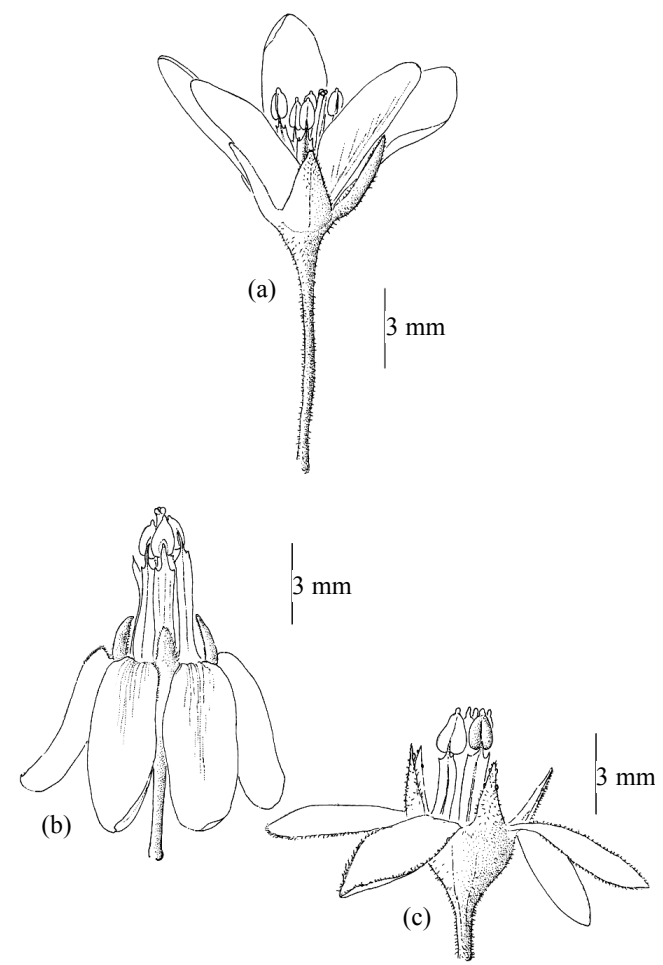

Figure 9. Flowers of Pterostemon. A. P. bravoanus (from Carreto 678 at FCME). B. P. rotundifolius (from Vega 3 at FCME). C. P. mexicanus (from Cruz 5998 at FCME). Drawings R. Cruz.

1) Leaves elliptic, oblong-orbiculate, suborbiculate or orbiculate, petiole $0.7-3.5 \mathrm{~cm}$ long; inflorescences usually with more than 20 flowers; Oaxaca, Guerrero, and Southern Puebla.

2) Inflorescences with less than 50 flowers; petals 7 $8 \mathrm{~mm}$ long, 3 - $4 \mathrm{~mm}$ wide, oblong, reflexed; Oaxaca and Southern Puebla …….. P. rotundifolius

2) Inflorescences with more than 50 flowers; petals 4 - $6.5 \mathrm{~mm}$ long, 2 - $2.5 \mathrm{~mm}$ wide, elliptic, erect; Guerrero …….................... P. bravoanus

\subsection{Description of Species}

\subsubsection{Pterostemon bravoanus J. Jiménez-Ram. \& Martínez Gordillo. Acta Bot. Mex. 41: 21-24.} 1997. TYPE: MEXICO. Guerrero: Mun. Eduardo Neri, 8 km NE of Carrizalillo, on Road to Mezcala, 7 Oct. 1996, Cruz \& García 975

(Holotype: FCME!; Isotypes: IEB!, MEXU!).

Shrubs or small trees, $1-8 \mathrm{~m}$ tall; cortex smooth, young branches pubescent. Leaves petiolated; petiole $1.4-3.5$ $\mathrm{cm}$ long, pubescent; stipules $1.5-2 \mathrm{~mm}$ long, linear, pubescent, persistent; blade $3.5-9 \mathrm{~cm}$ long, $4-7 \mathrm{~cm}$ wide, membranaceous, widely elliptic to orbicular, apex rounded, margin crenate-toothed, flat, base rounded to obtuse, adaxial surface glabrous, abaxial surface pubescent, secondary veins 5 - 6 pairs, the most proximal pair

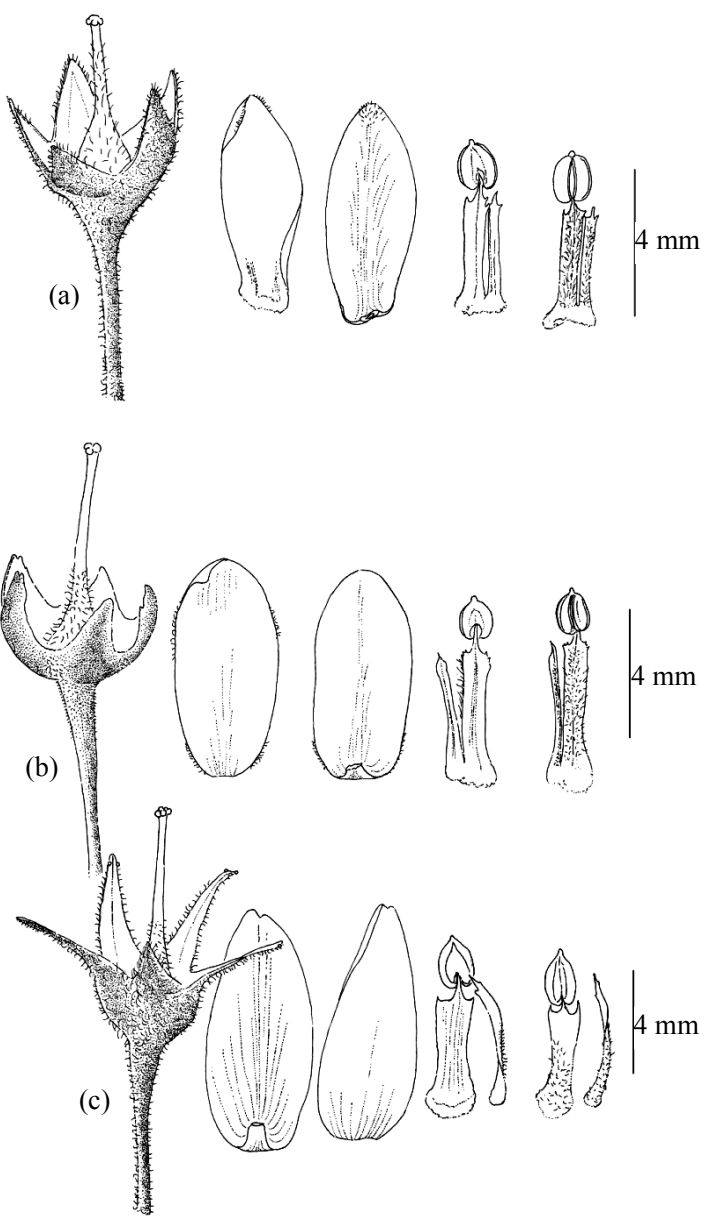

Figure 10. Floral parts of Pterostemon. A. P. bravoanus (from Cruz \& Ponce 5899 at FCME). B. P. rotundifolius (from Vega 3 at FCME). C. P. mexicanus (from Castrejón 1334 at FCME). Drawings R. Cruz.
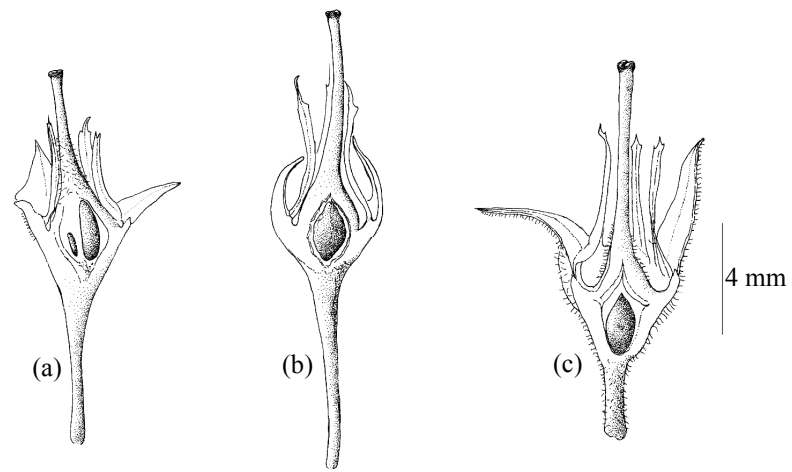

Figure 11. Fruits of Pterostemon. A. P. bravoanus (from Cruz \& Ponce 5899 at FCME). B. P. rotundifolius (from Vega 3 at FCME). C. P. mexicanus (from Cruz 5999 at FCME). Drawins R. Cruz.

suprabasal. Inflorescences 5 - $10 \mathrm{~cm}$ long, in subterminal cymes with 50 flowers or more, peduncle pubescent; bracteoles $2-2.5 \mathrm{~mm}$ long, linear, pubescent. Flowers pedicellate; pedicel 4 - $6 \mathrm{~mm}$ long; sepals 5, $1.3-3 \mathrm{~mm}$ 
long, 1.4 - $1.8 \mathrm{~mm}$ wide, deltate, apex acute, adaxial surface glabrous, abaxial surface pubescent; petals 5, 4-6.5 $\mathrm{mm}$ long, 2 - $2.5 \mathrm{~mm}$ wide, elliptic, apex rounded, base straight, white, erect, adaxial surface glabrous, abaxial surface pubescent; stamens 5, staminodia 5; filaments flattened, wide and winged, pubescent abaxially; anthers elliptic, apiculate; pollen tricolporate, spheroidal; exine tectate, perforated (Figure 12), bicellular when freed; ovary 5-locular, pubescent, 4 - 6 ovules per carpel; styles 5, pubescent, slightly free at the distal end; stigmas 5-lobed, free. Fruit an indehiscent capsule. Seed usually one per fruit, ovoid, maroon.

Phenology. Flowering from March to November.

Distribution and habitat. In Quercus forest and tropical deciduous forest between 1300 and $1600 \mathrm{~m}$ above sea level.

Additional Specimens Examined. MEXICO. GUERRERO: Mun. Ahuacotzingo, Ajuatetla, Peaseant Reserve, 14 Mar 1999, Casarrubias 44 (FCME); aprox. 20 $\mathrm{km} \mathrm{S}$ of San Juan Las Joyas, on road to Chilapa, $17^{\circ} 40^{\prime} 11^{\prime \prime} \mathrm{N}, 99^{\circ} 3^{\prime} 54^{\prime \prime} \mathrm{W}, 1440$ m, 28 Jul 1978, Martínez 308 (FCME, MEXU); Mun. Atlixtac, Petatlán, $17^{\circ} 36^{\prime} 0^{\prime \prime} \mathrm{N}, 99^{\circ} 0^{\prime} 0^{\prime \prime} \mathrm{W}, 1,579 \mathrm{~m}, 18$ Aug 2004, Carreto 678 (FCME); $3.8 \mathrm{NW}$ of Petatlán, $17^{\circ} 36^{\prime} 15^{\prime \prime} \mathrm{N}$, 99 $9^{\circ} 4.6^{\prime \prime} \mathrm{W}, 1300$ m, 9 Nov. 2000, Cruz 5558 (FCME); Mun. Chilpancingo, Chilpancingo de los Bravo, $5 \mathrm{mi}$ $\mathrm{W}$, on road to Omiltemi, $17^{\circ} 33^{\prime} 54.44^{\prime \prime} \mathrm{N}, 99^{\circ} 33^{\prime} 12.45^{\prime \prime} \mathrm{W}$, 1,900 m, 21 Oct. 1962, McVaugh 21916 (ENCB, TEX); Salto de Valadez, $122 \mathrm{~km} \mathrm{~S}$ of Chilpancingo, $17^{\circ} 26^{\prime} 0^{\prime \prime} \mathrm{N}$, 99²7'40"W, 1360 m, 7 Aug 1976, Patiño s.n. (IEB, MEXU, ENCB); Mun. Eduardo Neri, Zopilote canyon, Oct. 1932, Bravo-Hollis 3223 (MEXU); Xochipala, 5.5 $\mathrm{km}$ NW, on road to Filo de Caballo, $17^{\circ} 54^{\prime} \mathrm{N}, 99^{\circ} 39^{\prime} \mathrm{W}$, Cruz \& Ponce 5899 (FCME); Mezcala, $11.6 \mathrm{~km}$ al SO, $17^{\circ} 51^{\prime} 35^{\prime \prime} \mathrm{N}, 99^{\circ} 40^{\prime} 20^{\prime \prime} \mathrm{W}, 1495 \mathrm{~m}, 6$ Sep 2000, Cruz \& Castrejón 4983 (FCME); 8 km NE of highway Mexico-Acapulco, on road to Huiziltepec, 10 Nov. 1993, García 88 (FCME); $10 \mathrm{~km}$ of Mezcala, towards Carrizalillo, $17^{\circ} 50^{\prime} 34^{\prime \prime} \mathrm{N}, 99^{\circ} 36^{\prime} 3^{\prime \prime} \mathrm{W}, 1540$ m, 24 Nov. 1994, Martínez 1012 (FCME).

\subsubsection{Pterostemon mexicanus Schauer, Linnaea} 20:726. 1847. TYPE: MEXICO. Hidalgo: Crescit in Montosis Mexici, ad Zimapan, Aschb. 259 (Holotype: Herbarium Unknown)

Shrubs, 1 - $3 \mathrm{~m}$ tall; cortex ribbed, young branches pubescent. Leaves petiolate; petiole 4 - $6 \mathrm{~mm}$ long, pubescent; stipules 0.5 - $1 \mathrm{~mm}$ long, linear-subulate pubescent, eciduous; blade (0.7-)2 - $4 \mathrm{~cm}$ long, 1.2 - $3 \mathrm{~cm}$ wide, subcoriaceous, obovate to widely obovate; apex obtuse, margin dentate or double dentate in the upper $2 / 3$, revolute, base cuneiform, adaxial surface pilose, adaxial surface densely pubescent, secondary veins $3-4$ pairs, proximal pair suprabasal. Inflorescences 1.5 - $2 \mathrm{~cm}$ long,

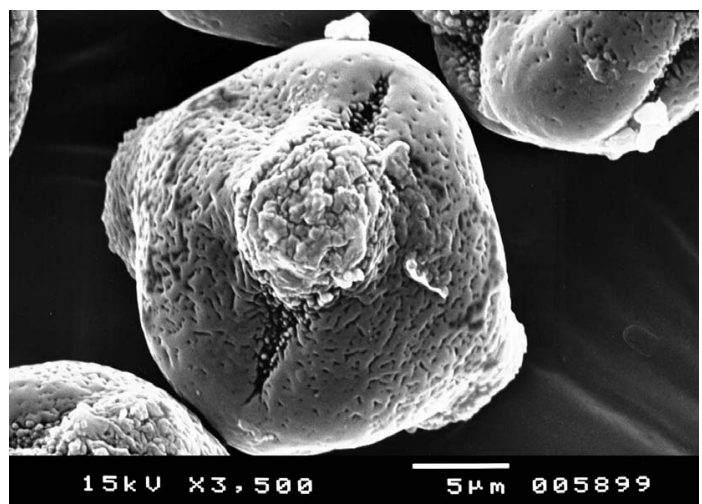

Figure 12. Pollen of Pterostemon bravoanus, equatorial view (from Cruz \& Ponce 5899 at FCME).

in subterminal cymes, with 5 - $12(-20)$ flowers, peduncles pubescent; bracteoles 1.5 - $2 \mathrm{~mm}$ long, linearlanceolate, pubescent. Flowers pedicelled, pedicels 4 - 5 mm, pilose; sepals 5, (3-)4 - $5 \mathrm{~mm}$ long, $1.5 \mathrm{~mm}$ wide, linear-lanceolate, apex acuminate; adaxial surface glabrous, abaxial surface pubescent; petals 5, 8 - $10 \mathrm{~mm}$ long, 4 - $5 \mathrm{~mm}$ wide, oblong, apex emarginated, base unguiculate, white, slightly reflexed, adaxial surface glabrous, abaxial surface pilose; stamens 5, staminodia 5; filaments flattened, wide and winged, pubescent on the abaxial surface; anthers cordiform, apiculated; pollen tricolporoidate, prolate, with the transversal colpi circular, exine tectate perforated, and suprarrugulated (Figures 13, 14), tricellular when freed; ovary 5 locular, pubescent, 4 - 8 ovules per carpel; styles 5, pubescent, free at the distal end; stigmas 5, lobulated, sometimes free. Fruit an indehiscent capsule. Seeds generally one per fruit, ovoid, maroon.

Phenology. Flowering throughout the year.

Distribution and habitat. In dry scrublands and Pinus forest, in altitudes between 500 and $2350 \mathrm{~m}$.

Additional Specimens Examined. MEXICO. GUANAJUATO: Mun. Atarjea, Manga Cuata, $8 \mathrm{~km} \mathrm{~N}$ of Atarjea, $21^{\circ} 15^{\prime} 30^{\prime \prime} \mathrm{N}, 99^{\circ} 45^{\prime} 42^{\prime \prime} \mathrm{W}, 1,300 \mathrm{~m}, 16$ Nov. 1989, Ventura \& López 7666(ENCB, IEB, MEXU, XAL); Mun. Xichú, La Aurora mine, $6 \mathrm{~km}$ E of Xichú, $21^{\circ} 17^{\prime} 99^{\prime \prime} \mathrm{N}, 100^{\circ} 3^{\prime} 99^{\prime \prime} \mathrm{W}, 1,200$ m, 30 Oct. 1986, Rzedowski 41511 (ENCB, MEXU, IEB, XAL); La Cuchilla hill, $15 \mathrm{~km} \mathrm{~N}$ of Xichú, $21^{\circ} 17^{\prime} 99^{\prime \prime} \mathrm{N}, 10^{\circ} 3^{\prime} 99^{\prime \prime} \mathrm{W}$, 7 Dic 1988, 1,800 m, Ventura \& López 6432 (ENCB, IEB, MO, XAL). HIDALGO: Mun. Cardonal, Tolantongo, La Piedra Cerrada canyon, $20^{\circ} 18^{\prime} \mathrm{N}, 99^{\circ} 00^{\prime} \mathrm{W}$, 2,100 m, 28 Aug 1976, González, Ortiz, Hiriart \& Solís 9468, 9509 (ENCB, MEXU, MO); Tolantongo canyon, at bottom, $20^{\circ} 45^{\prime} 24^{\prime \prime} \mathrm{N}, 99^{\circ} 1^{\prime} 42^{\prime \prime} \mathrm{W}, 1300 \mathrm{~m}, 16$ Oct. 1983, Rzedowski 38345 (ENCB, IEB, MEXU); Mun. Ixmiquilpan, $3 \mathrm{~km} \mathrm{SE}$ of $\mathrm{El} \mathrm{Cubo,} \mathrm{road} \mathrm{to} \mathrm{Arroyo}$ Hondo, 20 $37^{\prime} 55.2^{\prime \prime} \mathrm{N}, 99^{\circ} 01^{\prime} 36.8^{\prime \prime} \mathrm{W}, 1,945 \mathrm{~m}, 20 \mathrm{Mar}$ 1999, Castrejón 509 (FCME), 28 Oct. 2000, 1334 


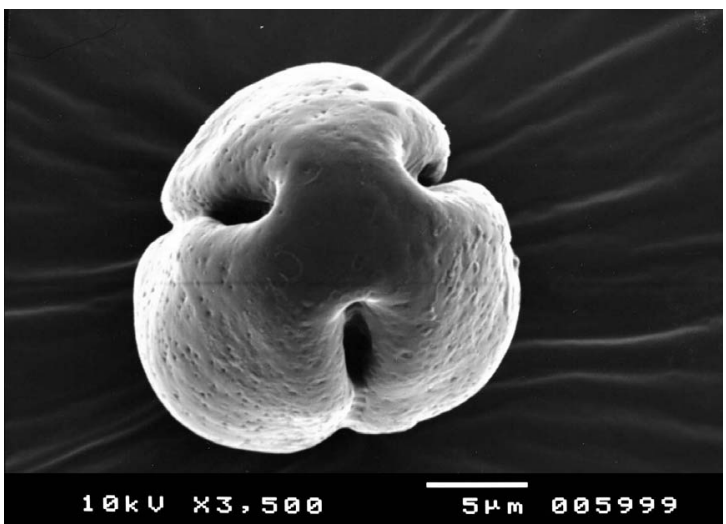

Figure 13. Pollen of Pterostemon mexicanus, polar view (from Cruz 5999 at FCME).

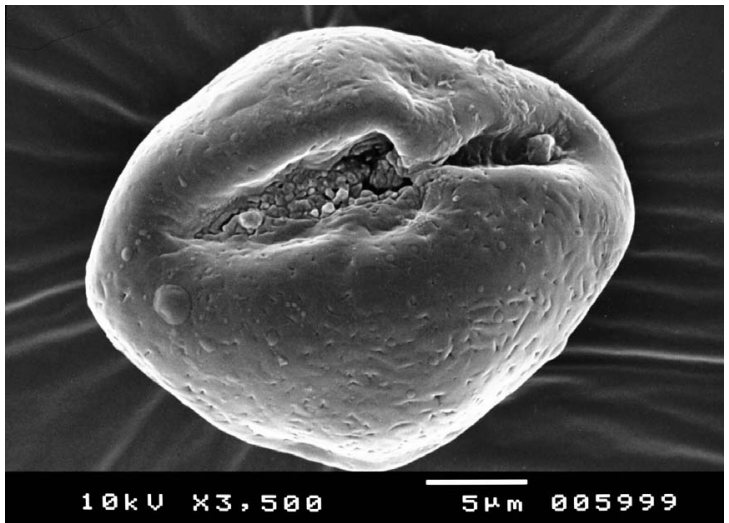

Figure 14. Pollen of Pterostemon mexicanus, equatorial view (from Cruz 5999 at FCME).

(FCME); El Cubo, 3 km SE, Tolantongo canyon, 1,900 m, Cruz 5998, 5999 (FCME); Mun. Jacala de Ledezma, Jacala de Ledezma, 2059'48"N, 99 11'33"W, Apr 1953, Paray 355 (ENCB); Mun. Metztitlán, $3 \mathrm{~km} \mathrm{~W}$ of Meztitlán, road to San Pablo, 20³7'22"N, 98 52'53"W, 22 Oct. 2000, Miranda 1039 (MEXU); Mun. Zimapán, Río Tolimán canyon, $20^{\circ} 48^{\prime} 30^{\prime \prime} \mathrm{N}, 99^{\circ} 26^{\prime} 49^{\prime \prime} \mathrm{W}, 1,000$ m, 11 Jun 1993, Zamudio \& Pérez-Cálix 9121 (MEXU). QUERETARO: Mun. Cadereyta de Montes, La Tinaja, road to San Joaquín, Balderas \& Serrano 23 (MEXU); 8 $\mathrm{km} \mathrm{N}$ of Vizarrón, El Mirador, 20 $52^{\prime} 48^{\prime \prime} \mathrm{N}, 99^{\circ} 42^{\prime} 40^{\prime \prime} \mathrm{W}$, 1,900 m, 10 Nov. 1984, Fernández 2623 (ENCB, IEB MEXU); Mun. Peñamiller, $5 \mathrm{~km} \mathrm{NW}$ of Peñamiller, on road to Aldama, $21^{\circ} 5^{\prime} 9^{\prime \prime} \mathrm{N}, 99^{\circ} 49^{\prime} 13.67^{\prime \prime} \mathrm{W}, 1,580 \mathrm{~m}, 9$ Nov. 1977, Zamudio 2544 (MEXU, IEB, ENCB, TEX, XAL); Mun. Pinal de Amoles, SSO of Cuatro Palos, $21^{\circ} 8^{\prime} \mathrm{N}, 99^{\circ} 33^{\prime} \mathrm{W}, 2,000$ m, 12 Jan 1990, Carranza 2304 (ENCB, IEB, MEXU, XAL); Mun. San Joaquín, La Culebra canyon, aprox. $5 \mathrm{~km}$ NE of La Tinaja, 20 Oct. 1994, Zamudio 9407 (MEXU, IEB). SAN LUIS POTOSI: Mun. Lagunillas: $3 \mathrm{~km}$ SE of Lagunillas, $21^{\circ} 34^{\prime} 3^{\prime \prime} \mathrm{N}, 99^{\circ} 32^{\prime} 47.92^{\prime \prime} \mathrm{W}, 850 \mathrm{~m}, 29$ Apr 1956, Rzedowski 7622 (MEXU, TEX).

\subsubsection{Pterostemon rotundifolius Ramírez, Estudio 4: 453. pl. 18. 1893. Type: MEXICO. Oaxaca: between Jaltepetongo and Guandulain, 1891/1, Altamirano H. S.n. (Holotype: US)}

Shrubs, 1.5 - $3 \mathrm{~m}$ tall; cortex ribbed, young branches pubescent. Leaves petiolate, petioles $7-9.5 \mathrm{~mm}$ long, pubescent; stipules 1.1 - $2 \mathrm{~mm}$ long, linear, pubescent, deciduous; blade 2 - $3.2 \mathrm{~cm}$ long, 2.2 - $3.3 \mathrm{~cm}$ wide, coriaceous, suborbicular, oblong-orbicular, sometimes slightly oblong, apex obtuse, margin dentate-crenate, generally revolute, base rounded or slightly subcordate; adaxial surface glabrous, abaxial surface pilose or glabrous, secondary veins $4-5$ pairs, the proximal pair basal. Inflorescences $2-2.6 \mathrm{~cm}$ long, in terminal cymes, with 9 - 22 or less than 50; peduncles puberulent; bracteoles 2 - $2.5 \mathrm{~mm}$ long, linear, pubescent. Flowers pedicellate, pedicels 3.5 - $5 \mathrm{~mm}$ long; sepals 5, $2.5 \mathrm{~mm}$ long, deltate-lanceolate, apex acute, adaxial and abaxial surfaces pilose; petals 5, 7 - $8 \mathrm{~mm}$ long, 3 - $4 \mathrm{~mm}$ wide, oblong, apex rounded, base slightly cuneiform, pink, white when old, reflexed, adaxial and abaxial surfaces pilose; stamens 5, staminodia 5; filaments flattened, wide and winged, pubescent adaxially; anthers cordiform, apiculated; pollen tricolporated, spheroidal, with the transversal colpi circular; exine tectated, perforated (Figures 15, 16), tricellular when freed; ovary 5-locular, pubescent; 4 - 8 ovules per carpel; styles 5 , hispid in the proximal half, connate throughout; stigmas 5-lobed, connate to slightly free. Fruit an indehiscent capsule. Seeds generally one per fruit, ovoid, maroon.

Phenology.- Flowering from January to September.

Distribution and habitat.- In dry scrublands or Quercus/Brahea forest, from 500 to 1900 meters above sea level.

Additional Specimens Examined.-MEXICO. OAXACA: Mun. Asunción Nochixtlán, $89 \mathrm{~km}$ by road $\mathrm{S}$ of Teotitlán del Camino on road to Oaxaca, $17^{\circ} 34^{\prime} 0^{\prime \prime} \mathrm{N}$, $96^{\circ} 57^{\prime} 0^{\prime \prime} \mathrm{W}, 1500 \mathrm{~m}, 10$ Oct. 1983, Anderson 12986 (MEXU); $50 \mathrm{~km} \mathrm{~N}$ of San Francisco Telixtlahuaca, road to Tehuacán, $17^{\circ} 33^{\prime} 0^{\prime \prime} \mathrm{N}, 96^{\circ} 58^{\prime} 0^{\prime \prime} \mathrm{W}, 1,524 \mathrm{~m}, 6$ Nov. 1983, Breedlove 59835 (MEXU); Concepción Buenavista, $0.5 \mathrm{~km}$ on road $\mathrm{km} 109$ highway TehuacánOaxaca, $18^{\circ} 01^{\prime} 11.3^{\prime \prime} \mathrm{N}, 97^{\circ} 21^{\prime} 21.2^{\prime \prime} \mathrm{W}, 1,590 \mathrm{~m}$, Téllez 17066 (FCME); Mun. Heroica Ciudad de Huajuapan de León, Santiago Miltepec, $17 \mathrm{~km}$ al NE de Miltepec, $48 \mathrm{~km}$ NE of Huajuapan de León, road to Tehuacán, $17^{\circ} 59^{\prime} 0^{\prime \prime} \mathrm{N}, 97^{\circ} 41^{\prime} 0^{\prime \prime} \mathrm{W}, 4$ Sep 1979, Chiang 406 (XAL); Huajuapan de León, $17^{\circ} 48^{\prime} 9^{\prime \prime} \mathrm{N}, 97^{\circ} 46^{\prime} 34^{\prime \prime} \mathrm{W}, 1,475 \mathrm{~m}$, 10 Jul 1994, Panero 4153 (IEB, TEX); La Loma Pachona, $6 \mathrm{~km} \mathrm{NW}$ of Guadalupe Cuautepec, $1 \mathrm{~km}$ E of crossection road Huajuapan de León-Tehuacán, $18^{\circ} 1^{\prime} 0^{\prime \prime} \mathrm{N}$, $97^{\circ} 39^{\prime} 0^{\prime \prime} \mathrm{W}, 2,060$ m, 27 Nov. 1986, Salinas F-3688 (MEXU); Mun. Miahuatlán de Porfirio Díaz, road to Quixtla, $16^{\circ} 15^{\prime} 48^{\prime \prime} \mathrm{N}, 96^{\circ} 35^{\prime} 36^{\prime \prime} \mathrm{W}, 1,900 \mathrm{~m}, 13$ Jun 


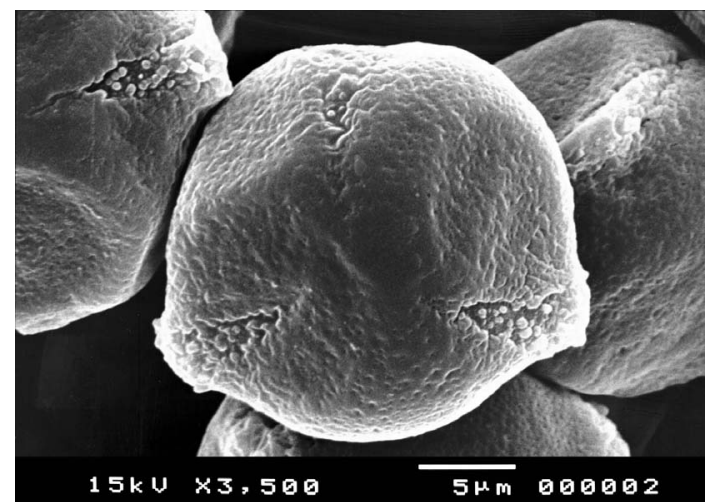

Figure 15. Pollen of Pterostemon rotundifolius, polar view (from Vega 2 at FCME).

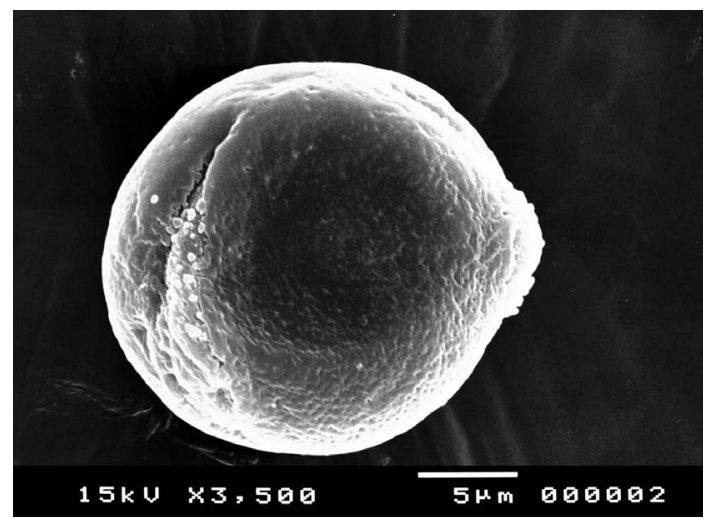

Figure 16. Pollen of Pterostemon rotundifolius, equatorial view (from Vega 2 at FCME).

1985, García-Mendoza 1600 (MEXU); Miahuatlán de Porfirio Díaz, $16^{\circ} 15^{\prime} 48^{\prime \prime} \mathrm{N}, 96^{\circ} 35^{\prime} 36^{\prime \prime} \mathrm{W}, 25$ Oct. 1982,

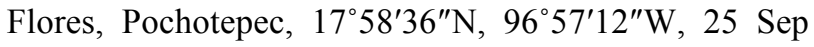
1990, Tenorio 17208 (IEB); Mun. San Antonio Nanahuatipam, $10 \mathrm{~km} \mathrm{~S}$ of Ignacio Mejia, Río Xiquila, $18^{\circ} 2^{\prime} 0^{\prime \prime} \mathrm{N}, 97^{\circ} 11^{\prime} 0^{\prime \prime} \mathrm{W}, 990 \mathrm{~m}, 16$ Nov. 1987, Salinas 4535 (MEXU, XAL); Mun. Oaxaca de Juárez, Las Hoyas canyon, $17^{\circ} 19^{\prime} 0^{\prime \prime} \mathrm{N}, 96^{\circ} 56^{\prime} 0^{\prime \prime} \mathrm{W}, 1,500 \mathrm{~m}, 2$ Nov. 1994, Pringlei 6027 (ENCB); Mun. San Andrés Sinaxtla, cañada NW of Santa María Ixcatlán, $17^{\circ} 51^{\prime} 0^{\prime \prime} \mathrm{N}$, $97^{\circ} 11^{\prime} 0^{\prime \prime} \mathrm{W}, 2,200$ m, 10 May 1992, Tenorio 18340 (TEX); Mun. San Francisco Telixtlahuaca, San Francisco Telixtla- huaca; $50 \mathrm{~km} \mathrm{~N}$, along highway $131,17^{\circ} 35^{\prime} 3^{\prime \prime} \mathrm{N}$, $96^{\circ} 56^{\prime} 53^{\prime \prime} \mathrm{W}, 1,524$ m, 6 Nov. 1983, Breedlove 59835 (TEX); Mun. San Juan Bautista Cuicatlán, 11 km NE of Cuicatlán, $17^{\circ} 32^{\prime} 0^{\prime \prime} \mathrm{N}, 96^{\circ} 57^{\prime} 0^{\prime \prime} \mathrm{W}, 1,400 \mathrm{~m}, 29$ Aug 1980, González F-1741 (MEXU); San Jerónimo Sosota, El Parian-Las Sedas [San Sebastián Sedas], km 313 - 314 of rail road, $17^{\circ} 23^{\prime} 0^{\prime \prime} \mathrm{N}, 97^{\circ} 0^{\prime} 0^{\prime \prime} \mathrm{W}, 1,500 \mathrm{~m}, 29$ May 1992, Salinas 6864 (MEXU); Mun. San Marcos Arteaga: $30.5 \mathrm{~km}$ SO of Huajuapan de León, $8 \mathrm{~km}$ SO of San Marcos Arteaga, road to Tonalá, $17^{\circ} 44^{\prime} \mathrm{N}, 97^{\circ} 56^{\prime} \mathrm{W}$, 1,750 m, 10 Feb 1993, Campos 5017 (MEXU); Mun. San Mateo Tlapiltepec, $4 \mathrm{~km}$ NE of La Unión, $17^{\circ} 56^{\prime} 0^{\prime \prime}$
N, $97^{\circ} 21^{\prime} 0^{\prime \prime} \mathrm{W}, 2300$ m, 9 Oct. 1970, Cruz 2641 (ENCB); Mun. San Pedro Jaltepetongo, road from San Pedro Jaltepetongo towards Tomellín, km 6 SE, $17^{\circ} 44^{\prime} 25^{\prime \prime} \mathrm{N}$, $96^{\circ} 59^{\prime} 10^{\prime \prime} \mathrm{W}, 1,475 \mathrm{~m}, 11$ Jun 2002, Medina 1096 (MEXU); $14.3 \mathrm{~km}$ of Tomellín, towards Santa María Texcatitlán, $17^{\circ} 43^{\prime} 06^{\prime \prime} \mathrm{N}, 97^{\circ} 01^{\prime} 27.1^{\prime \prime} \mathrm{W}, 29$ Jun 2004 , Téllez 16063 (FCME); $23 \mathrm{~km}$ of Tomellín, towards Santa María Texcatitlán, $17^{\circ} 42^{\prime} 58.4^{\prime \prime} \mathrm{N}, 97^{\circ} 01^{\prime} 28.3^{\prime \prime} \mathrm{W}$, Téllez 16079 (FCME); Mun. San Pedro Jocotipac, Cuicatlán, aprox. $20 \mathrm{mi} \mathrm{S}, 17^{\circ} 35^{\prime} 0^{\prime \prime} \mathrm{N}, 96^{\circ} 55^{\prime} 0^{\prime \prime} \mathrm{W}, 1,200 \mathrm{~m}, 23 \mathrm{Aug}$ 1975, Webster 20152 (IEB, MEXU); Mun. Santa María Apazco, $18^{\circ} 12^{\prime} 57.5^{\prime \prime} \mathrm{N}, 97^{\circ} 38^{\prime} 46.8^{\prime \prime} \mathrm{W}$, Téllez 16389 (MEXU); Mun. Santa María Ixcatlán, towards Santa

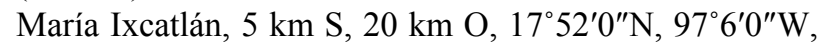
15 Sep 1991, Salinas 6109 (MEXU); Tenorio 17841 (MEXU); Mun. Santa María Tecomavaca: Barranca seca, road to Santa María Ixcatlán, $12 \mathrm{~km} \mathrm{W,} 17^{\circ} 56^{\prime} 0^{\prime \prime} \mathrm{N}$, 97³'0"W, 19 Nov. 1991, Tenorio 18118 (MEXU);

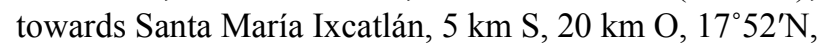
976' W, 15 Sep 1991, Salinas 6109 (MEXU); Mun. Santiago Chazumba, $4 \mathrm{~km}$ NE of Santiago Chazumba, highway Huajuapan de León-Tehuacán, $18^{\circ} 13^{\prime} 0^{\prime \prime} \mathrm{N}$, $97^{\circ} 41^{\prime} 0^{\prime \prime} \mathrm{W}, 2,000 \mathrm{~m}, 22$, Mar 1980, González F-954 (MEXU, TEX); $2 \mathrm{~km} \mathrm{~S}$ limits of Oaxaca and Puebla, highway Huajuapan de León-Tehuacán, $18^{\circ} 13^{\prime} 0^{\prime \prime} \mathrm{N}$, 9741'0"W, 27 Jul 1979, Chiang F-188 (MEXU); Mun. Santiango Nacaltepec, Jayacatlán, north along road towards Nacaltepec, $17^{\circ} 30^{\prime} 9^{\prime \prime} \mathrm{N}, 96^{\circ} 55^{\prime} 11^{\prime \prime} \mathrm{W}, 1,600 \mathrm{~m}, 4$ Nov. 1973, Breedlove 35942 (ENCB); Mun. Santiago Tenango, Las Hoyas canyon, $17^{\circ} 19^{\prime} 0^{\prime \prime} \mathrm{N}, 96^{\circ} 56^{\prime} 0^{\prime \prime} \mathrm{W}$, 1,500 m, 2 Nov. 1894, Pringlei 6027 (MEXU); Mun. San Juan Bautista Suchitepec, La Loma Pachona, 6 km NE of Guadalupe Cuautepec or $1 \mathrm{~km}$ E of junction highway to Huajuapan de León-Tehuacán and road to Guadalupe Cuautepec; $18^{\circ} 27^{\prime} 0^{\prime \prime} \mathrm{N}, 97^{\circ} 36^{\prime} 0^{\prime \prime} \mathrm{W}, 27$ Nov. 1986, Salinas 3688 (IEB, XAL); Mun. Teotitlán del Valle, $89 \mathrm{~km}$ by road $\mathrm{S}$ of Teotitlán del Camino, on road to Oaxaca, $17^{\circ} 34^{\prime} 0^{\prime \prime} \mathrm{N}, 96^{\circ} 57^{\prime} 0^{\prime \prime} \mathrm{W}, 1,500 \mathrm{~m}, 10$ Oct. 1983, Anderson 12986 (IEB); Flores Magón, 10 km S of Ignacio Mejía, Río Xiquila, Tierra Grande 2 - $3 \mathrm{~km} \mathrm{E,} 18^{\circ} 2^{\prime} 0^{\prime \prime} \mathrm{N}$, $97^{\circ} 11^{\prime} 0^{\prime \prime} \mathrm{W}, 850$ m, 16 Nov. 1987, Salinas 4535 (IEB); Santa María Tecomavaca, $17^{\circ} 51^{\prime} 0^{\prime \prime} \mathrm{N}, 97^{\circ} 8^{\prime} 0^{\prime \prime} \mathrm{W}$, Salinas 5853 (MEXU); Mun. Tepelmeme Villa de Morelos, Tepelmeme Villa de Morelos, $17^{\circ} 55^{\prime} 5^{\prime \prime} \mathrm{N}, 97^{\circ} 19^{\prime} 2.8^{\prime \prime} \mathrm{W}$, 2,350 m, 24 Aug 1968, Cruz 2131 (ENCB, TEX); 4 km NE of La Unión, $17^{\circ} 56^{\prime} 0^{\prime \prime} \mathrm{N}, 97^{\circ} 21^{\prime} 0^{\prime \prime} \mathrm{W}, 2,300 \mathrm{~m}, 9$ Oct. 1970, Cruz-Cisneros 2641 (ENCB, MEXU); Mun. San Pedro and San Pablo Tequixtepec, $17 \mathrm{~km}$ N of Santiago Miltepec, $48 \mathrm{~km}$ NE of Huajuapan de León-Tehuacán, $18^{\circ} 6^{\prime} 0^{\prime \prime} \mathrm{N}, 97^{\circ} 41^{\prime} 0^{\prime \prime} \mathrm{W}, 4$ Nov. 1979, Chiang F-406 (MEXU); Mun. Valerio Trujano, $1.8 \mathrm{~km}$ S of Tomellín, towards Nochistlán, $17^{\circ} 44^{\prime} 40.2^{\prime \prime} \mathrm{N}, 96^{\circ} 58^{\prime} 10.7^{\prime \prime} \mathrm{W}, 29$ Jun 2004, Téllez 15974 (FCME). PUEBLA: Mun. Atexcal, Guadalupe hill, NW of San Martín Atexcal, 
$18^{\circ} 26^{\prime} 58^{\prime \prime} \mathrm{N}, 97^{\circ} 40^{\prime} 56^{\prime \prime} \mathrm{W}, 1,870 \mathrm{~m}, 15$ Oct. 1984, Tenorio 7693 (ENCB, MEXU); Mun. Atoyatempan, Tepeyehualco, $9 \mathrm{~km} \mathrm{SE}$, Cascadas of Acatzitzimitla, Atoyatempan, $18^{\circ} 45^{\prime} 7 \mathrm{~N}, 97^{\circ} 53^{\prime} 18^{\prime \prime} \mathrm{W}, 1,890 \mathrm{~m}, 17$ Nov. 1984, Fernández 2640 (ENCB, MEXU); Mun. Caltepec, La Huerta barranca, NE of Caltepec, $18^{\circ} 10^{\prime} 53^{\prime \prime} \mathrm{N}$, $97^{\circ} 28^{\prime} 45^{\prime \prime} \mathrm{W}, 1,890 \mathrm{~m}, 28$ May 1984, Tenorio 5938 (MEXU, TEX); El Coatepec hill, SE of Caltepec (PueblaCaltepec) $18^{\circ} 9^{\prime} 7^{\prime \prime} \mathrm{N}, 97^{\circ} 26^{\prime} 43^{\prime \prime} \mathrm{W}, 2100 \mathrm{~m}, 11$ Aug 1984, Tenorio 6815 (TEX); Mun. San Francisco Chapulapa, $1.6 \mathrm{~km}$ of road on $\mathrm{km} 25.8$, highway Coacnolapan-Oaxaca, $18^{\circ} 37^{\prime} 0.35^{\prime \prime} \mathrm{N}, 97^{\circ} 28^{\prime} 10.9^{\prime \prime} \mathrm{W}, 27$ Oct. 2005, Rosas 364 (FCME); Mun. San Antonio Cañada, $6 \mathrm{~km}$ E of San Antonio Cañada, $18^{\circ} 30^{\prime} 0^{\prime \prime} \mathrm{N}, 97^{\circ} 16^{\prime} 0^{\prime \prime} \mathrm{W}, 2,000 \mathrm{~m}, 20 \mathrm{Jul}$ 1990, Salinas 5507 (MEXU); behind cerro Colorado, between San Esteban Nacoxcalco and San Antonio Cañada, along the barranca Los Mangos, $18^{\circ} 30^{\prime} 0^{\prime \prime} \mathrm{N}$, $97^{\circ} 18^{\prime} 0^{\prime \prime} \mathrm{W}, 1,000 \mathrm{~m}, 16 \mathrm{Jul}$ 1961, Smith 4079 (MEXU); 8 $\mathrm{km} \mathrm{N}$ of San Antonio Cañada, $18^{\circ} 31^{\prime} 0^{\prime \prime} \mathrm{N}, 97^{\circ} 16^{\prime} 0^{\prime \prime} \mathrm{W}, 19$ May 1986, Tenorio 11302 (MEXU); Mun. Santiago Miahuatlán. Santiago Miahuatlán, $18^{\circ} 32^{\prime} 54^{\prime \prime} \mathrm{N}, 97^{\circ} 26^{\prime}$ $18^{\prime \prime} \mathrm{W}, 1400 \mathrm{~m}, 28$ Jul 1987, Martínez 21736 (IEB, MEXU); 6 - $7 \mathrm{~km}$ SO of San José Axusco, $18^{\circ} 13^{\prime} 0^{\prime \prime} \mathrm{N}$, $97^{\circ} 15^{\prime} 0^{\prime \prime} \mathrm{W}, 1,200 \mathrm{~m}, 18$ Aug 1988, Salinas 4794 (MEXU); Tlacuilosto barranca, S of San Juan Atzingo, $18^{\circ} 17^{\prime} 0^{\prime \prime} \mathrm{N}$, $97^{\circ} 24^{\prime} 0^{\prime \prime} \mathrm{W}, 1,900 \mathrm{~m}, 22$ Jul 1985, Tenorio 9440 (MEXU); Mun. Tehuacán, Tehuacán, $18^{\circ} 28^{\prime} 0^{\prime \prime} \mathrm{N}, 97^{\circ} 24^{\prime} 0^{\prime \prime} \mathrm{W}$, Sep 1911, Purpus 1382 (MEXU); 7.9 km from highway Fco. I. Madero-Tehuacán, entrance at Meseta San Lorenzo, $18^{\circ} 26^{\prime} 14.9^{\prime \prime} \mathrm{N}, 97^{\circ} 28^{\prime} 03.1^{\prime \prime} \mathrm{W}$, Téllez 19563 (MEXU); Santiago Acatepec, $18^{\circ} 13^{\prime} 36^{\prime \prime} \mathrm{N}, 97^{\circ} 34^{\prime} 42^{\prime \prime} \mathrm{W}, 2,120 \mathrm{~m}, 3$ Jul 1984, Tenorio 6649 (IEB, MEXU); Santiago Acatepec, $18^{\circ} 13^{\prime} 36^{\prime \prime} \mathrm{N}, 97^{\circ} 34^{\prime} 42^{\prime \prime} \mathrm{W}, 2,000 \mathrm{~m}, 25$ Jul 1978, Ventura 15399 (ENCB, IEB, MEXU); Mun. Tepeji de Rodríguez, 2 km SO of Tepeji de Rodríguez, 6 Jan 1981, GonzálezMedrano BC-68 (FCME); $12 \mathrm{~km} \mathrm{~W}$ of Molcaxac, $5 \mathrm{Apr}$ 1882, González-Medrano 12367 (MEXU); Mun. Tepeyahualco, $9 \mathrm{~km} \mathrm{SE}$, Cascadas de Acatzitzimitla, Atoyatempan, $18^{\circ} 45^{\prime} 7^{\prime \prime} \mathrm{N}, 97^{\circ} 56^{\prime} 18^{\prime \prime} \mathrm{W}, 17$ Nov. 1984, Fernández 2640 (IEB); Mun. Vicente Guerrero, 8 km N of San Antonio Cañada, $18^{\circ} 33^{\prime} 0^{\prime \prime} \mathrm{N}, 97^{\circ} 16^{\prime} 12^{\prime \prime} \mathrm{W}, 2,030 \mathrm{~m}$, 19 May 1986, Tenorio 11302 (MEXU); Mun. Zapotitlán, $4 \mathrm{~km}$ NE of Santiago Acatepec, highway Huajuapan de León-Tehuacán, $18^{\circ} 15^{\prime} 0^{\prime \prime} \mathrm{N}, 97^{\circ} 33^{\prime} 0^{\prime \prime} \mathrm{W}$, 5 Sep 1979 , Chiang F-432 (MEXU); $8 \mathrm{~km}$ NE of Santiago Acatepec, $18^{\circ} 16^{\prime} 0^{\prime \prime} \mathrm{N}, 97^{\circ} 33^{\prime} 0^{\prime \prime} \mathrm{W}, 16$ May 1981, Chiang F-1973 (MEXU); $4 \mathrm{~km}$ E of San Francisco Xochiltepec, $18^{\circ} 15^{\prime} 0^{\prime \prime} \mathrm{N}$, $97^{\circ} 25^{\prime} 0^{\prime \prime} \mathrm{W}, 2,120 \mathrm{~m}, 30$ Jul 1983, Chiang F-2402 (MEXU, TEX); Zapotitlán de Las Salinas, $18^{\circ} 20^{\prime} 0^{\prime \prime} \mathrm{N}$, $97^{\circ} 27^{\prime} 0^{\prime \prime} \mathrm{W}, 27$ Oct. 1960, Gómez-Pompa 361 (MEXU); $\mathrm{km} 79$, on road from Coyotepec to Acatlán, $18^{\circ} 19^{\prime} 39.6^{\prime \prime} \mathrm{N}$, $97^{\circ} 49^{\prime 2} 27.8^{\prime \prime} \mathrm{W}, 1,697$ m, 3 Jul 2003, Vega \& Jiménez s.n., 2, 3 (FCME).

\section{Conclusion}

The genus Pterostemon Schauer is included into the Iteaceae, a paleo-endemic family restricted to Mexico. It comprises three species of woody shrubs or small trees in arid and semi-arid regions. Pterostemon bravoanus is distinguished by presenting leaves with elliptic to orbicular lamina, inflorescences with more than 50 flowers, petals erect at anthesis, pollen spheroidal, and their distribution is restricted to the state of Guerrero; $P$. mexicanus has leaves with obovate to widely-obovate lamina, inflorescences with fewer than 20 flowers, petals extended at anthesis, pollen prolate, and distribution in the states of Guanajuato, Queretaro, Hidalgo, San Luis Potosi, and Puebla; P. rotundifolius has leaves with suborbicular to oblong-orbicular lamina, inflorescences with 2 - 50 flowers, petals reflexed at anthesis, pollen spheroidal, and is restricted in distribution to the states of Oaxaca and Puebla. In all three species the fruit is an indehiscent capsule with a solitary seed.

\section{Acknowledgements}

We thank José Antonio Hernández and Alejandro Martínez Mena, Laboratorio de Microcine, Facultad de Ciencias, Universidad Nacional Autónoma de México for digitalization of figures, to Silvia Espinosa Matías, Laboratorio de Microscopía Electrónica de Barrido, Facultad de Ciencias, Universidad Nacional Autónoma de México the photographs with scanning electron microscope. To the herbaria ENCB, FCME, IEB, MEXU, MO, TEX and XAL for access revision of botanical specimens.

\section{REFERENCES}

[1] J. Rzedowski, "El endemismo de la Flora Fanerogámica Mexicana: Una Apreciación Analítica Preliminar," Acta Botanica Mexicana, Vol. 15, 1991, pp. 47-64.

[2] S. Schauer, Linnaea, Vol. 20, 1874, pp. 736.

[3] H. Baillon, "Historie des Plantes," Paris, 1872.

[4] A. Engler and K. Prantl, "Saxifragaceae," In: A. Engler and K. Prantl, Eds., Die Natürlichen Pflazenfamilien, 2nd Edition, Vol. 18a, Wilhelm Engelmann, Leipzig, 1928, pp. 74-226.

[5] G. Erdtman, "Pollen Morphology and Plant Taxonomy Angiosperms," Azlmquist and Wilksell, Estocolmo, 1952, pp. 539.

[6] G. K. Schulze-Menz, "Saxifragaceae," In: H. Melchior Ed., A. Engler's Syllabus der Pflanzentamilien, Berlín, 1964, pp. 201-206.

[7] A. Cronquist, "An Integrated System of Classification of Flowering Plants," Columbia University Press, Nueva York, 1981, p. 1261.

[8] R. F. Thorne, "Classification and Geography of the Flowering Plants,” Botanical Review, Vol. 58, 1992, pp. 
225-348. http://dx.doi.org/10.1007/BF02858611

[9] J. K. Small, "Pterostemonaceae," In: N. L. Britton, et al., Eds., North American Flora, Vol. 22, The New York Botanical Garden, New York, 1905, p. 183.

[10] J. Hutchinson, "The Genera of Flowering Plants (Angiospermae)," Vol. I, Oxford at the Clarendon Press, Londres, 1967, p. 659.

[11] R. Dahlgren, "A Revised System of Classification of Angiosperms," Botanical Journal of the Linnean Society, Vol. 80, No. 2, 1983, pp. 91-124. http://dx.doi.org/10.1111/j.1095-8339.1980.tb01661.x

[12] A. Takhtajan, "Floristic Regions of the World," University of California Press, Berkeley, 1988, p. 522.

[13] E. Pérez-Cálix, "Pterostemonaceae," In: G. Calderón de Rzedowski and J. Rzedowski, Eds., Flora del Bajío y Regiones Adyacentes, Centro Regional del Bajío, Instituto de Ecología A.C., Pátzcuaro, 2003, p. 9.

[14] Angiosperm Phylogeny Group, APG III, “An Update of the Angiosperm Phylogeny Group Classification for the Orders and Families of Flowering Plants: APG III," Botanical Journal of the Linnean Society, Vol. 161, No. 4, 2009, pp. 105-121. doi: 10.1111/j.1095-8339.2009.00996

[15] D. E. Soltis, R. K. Kuzoff, M. E. Mort, M. Koontz and M. K. Arroyo, "Elucidating Deep-Level Phylogenetic Rela- tionships in Saxifragaceae Using Sequences for Six Chloroplast and Nuclear DNA Regions," Annals of the Missouri Botanical Garden, Vol. 88, No. 4, 2001, pp. 669-693. http://dx.doi.org/10.2307/3298639

[16] M. W. Chase, D. E. Soltis, R. G. Olmstead, D. Morgan, D. H. Les, B. D. Mishler, M. R. Duvall, R. Price, H. G. Hills, Y. Qui, K. Akron, J. H. Rettig, E. Conti, J. D. Palmer, J. R. Manhart, K. J. Sytsma, H. J. Michaels, W. J. Kress, K. G. Karol, W. D. Clark, M. Hedren, B. S. Gaut, R. K. Jansen, K. Kim, C. F. Wimpee, J. F. Smith, G. R. Furnier, S. H. Strauss, Q. Xiang, G. M. Plunkett, P. S. Soltis, S. M. Swernsen, S. E. Williams, P. A. Gadek, C. J. Quinn, L. E. Eguiarte, E. Golenberg, G. H. Learn Jr., S. Graham, S. C. H. Barrett, S. Dayanandan and V. A. Albert, "Phylogenetics of Seed Plants: An Analysis of Nucleotide Sequences from the Plastid Gene rbcL," Annals of the Missouri Botanical Garden, Vol. 80, No. 3, 1993, pp. 528-580. http://dx.doi.org/10.2307/2399846

[17] D. E. Soltis and P. S. Soltis, "Phylogenetic Relationships in Saxifragaceae Sensu Lato: A Comparison of Topologies Based on 18S rDNA," American Journal of Botany, Vol. 76, No. 8, 1997, pp. 1119-1124.

http://dx.doi.org/10.2307/2444824 